\title{
Approximation of elastic-plastic local strains in surface-hardened notched components
}

\author{
Patrick Yadegari ${ }^{1 *}$, Teresa Schlitzer ${ }^{2}$, and Michael Vormwald ${ }^{1}$ \\ ${ }^{1}$ Technical University of Darmstadt, Department of Civil and Environmental Engineering Sciences, \\ Materials Mechanics Group, 64287 Darmstadt, Germany \\ ${ }^{2}$ ihf Ingenieurgesellschaft mbH, 65835 Liederbach, Germany
}

\begin{abstract}
Elastic-plastic strains at points relevant for structural failures are usually approximated using formulas based on the stresses determined by elasticity theory. For this purpose, the Neuber approximation is a common method to estimate the local elastic-plastic strains in the notch root, although this is currently only approved for homogeneous components. For surfacehardened notched components, these approximation formulas need to be modified to cover two potential failure points: The notch root as well as the interface between the stronger surface layer and the weaker core material. In the following, a multi-step algorithm is shown that allows the estimation of elastic-plastic local strains at these two points, based on a single elasticitytheoretical solution. A comparison of the approximated values with those from finite element analyses (FEA) reveals that this results in only minor inaccuracies, while the usability is remarkable.
\end{abstract}

\section{Introduction}

Highly stressed components in mechanical engineering often require the use of surface layer hardening methods to ensure their resistance against wear and fatigue. The hardened surface layer then exhibits better mechanical properties than the core material, which results in a structure with inhomogeneous material properties. Furthermore, residual stresses have been introduced into the structure. While the elastic material properties of various areas do not differ significantly, the surface layer shows a considerably higher resistance to plastic deformation. Compared to a structure with homogeneous material properties, inhomogeneity only has an effect when the yield point is exceeded. Depending on the component and load introduction geometry, plastic deformation can first occur in the surface layer and in the area of the core material. In the case of thin surface layers, it is assumed that the surface layer neither contribute significantly to the load-carrying capacity nor noticeably influence the deformation behaviour of the component and that the core material's residual stress is negligible [1]. An application of this model succeeded in estimating suitable crack initiation lives compared to experimental results of notched specimens with residual stresses [2]. For thicker surface layers, which are in the magnitude of the notch radius, this approach may no longer be valid and can differ in practical application.

* Corresponding author: yadegari@wm.tu-darmstadt.de 
When performing proofs of structural durability based on the local strain approach, the elastic-plastic strains at the failure-relevant points, usually in the notch root, are estimated with approximation formulae based on the stresses determined by elasticity theory. For example, the "Guideline non-linear" of the Forschungskuratorium Maschinenbau (FKM) [3] allows the application of the Neuber formula, whereby this is only applicable for homogeneous components. To make the proof of structural durability based on the local strain approach also feasible for components reinforced by surface layers, this approximation methods must be extended to also cover the second failure-relevant point: The interface between the stronger surface layer and the weaker core material. A requirement for this is the identification of approximation formulas and algorithms, which can be used to estimate elastic-plastic stresses in such inhomogeneous structures. For this purpose, the results of nonlinear finite element analyses are used, whereby several different models were examined in order to determine the influence of the surface layer thickness $d$ and the notch radius $R$.

The analysed model is a surface-hardened round specimen with a circumferential notch under axial load. The hardness gradient is neglected for the approximation procedure and a strong simplification is introduced: The structure consists of only two, in itself homogeneous areas, namely the low strength core material and the high strength material of the surface layer, Figure 1. At the interface between these two areas exists deformation compatibility, whereby the equivalent strain of the core-side and surface-layer-side of the interface is also identical. For the finite element analyses only a two-dimensional section is used.

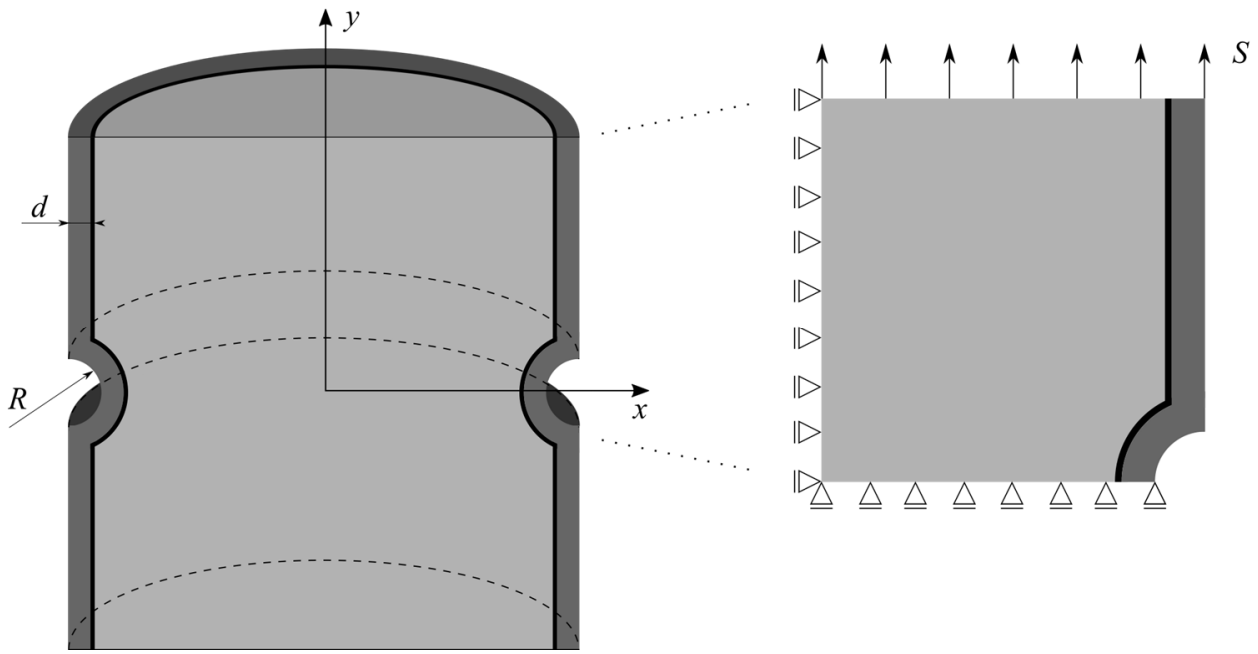

Fig. 1. Cross-section of a surface-hardened notched axisymmetric specimen with notch radius $R$ and surface layer thickness $d$. Two-dimensional section used for FEA with the boundary conditions shown.

\section{Approximation of elastic-plastic local strains}

For the approximation of elastic-plastic local strains based on elasticity-theoretical results, the Neuber method is commonly used. Since there is an additional failure-relevant point in surface-hardened components, the core-side interface, it is shown below that a modification of this procedure is required.

\subsection{Neuber's approximation method for the notch root}

The Neuber approximation method [4] allows the estimation of elastic-plastic local strains for the notch root on the basis of elasticity-theoretical calculations according to Equation 1. 
Since this equation cannot be solved explicitly, the stress is determined iteratively and the corresponding strain via the material law. Here, the procedure is extended to multi-axial stresses through the use of equivalent values for stress and strain [5].

$$
\sigma_{\mathrm{e}, \mathrm{V}} \cdot \varepsilon_{\mathrm{e}, \mathrm{V}} \cdot\left(\frac{\varepsilon^{*}}{\sigma^{*} / E}\right)=\sigma_{\mathrm{V}} \cdot \varepsilon_{\mathrm{V}}
$$

Furthermore an extended, more precise variant [6] of Neuber's approximation method is used, by including a modified strain function $\varepsilon^{*}$, which corresponds to the Ramberg-Osgood relationship, Equation 2. The therein included modified stress $\sigma^{*}$ allows a consideration of different notch geometries and types of load through the limit load factor $K_{\mathrm{p}}$. This factor is calculated via the quotient of the plastic load limit to the load at yield point, Equation 3.

$$
\begin{aligned}
& \varepsilon^{*}=\frac{\sigma^{*}}{E}+\left(\frac{\sigma^{*}}{K^{\prime}}\right)^{\frac{1}{n^{\prime}}} \quad \text { with } \quad \sigma^{*}=\frac{\sigma_{\mathrm{e}, \mathrm{V}}}{K_{\mathrm{p}}} \\
& K_{\mathrm{p}}=\frac{L_{\mathrm{p}}}{L_{\mathrm{F}}}
\end{aligned}
$$

\subsection{Modified approximation method for the interface}

In order to estimate the elastic-plastic strains for the core-side interface, the approximation method of Neuber has to be adapted. The first step is the definition of a virtual stress-strain relation between the surface layer and core material according to the Ramberg-Osgood relationship. This material model is calculated with a kind of mixing rule, taking into account the geometry-related influencing variables as the stress gradients in the notch root as well as the surface layer thicknesses $d$, Equation 4 .

$$
K_{\text {inter }}^{\prime}=K_{\text {core }}^{\prime}+\left(\frac{d}{1.5 r}\right) \cdot\left(K_{\text {surf.lay }}^{\prime}-K_{\text {core }}^{\prime}\right) \text { with } r=\frac{2 \cdot \sigma_{\mathrm{e}, \mathrm{V}, \text { notch root }}}{\left(\mathrm{d} \sigma_{\mathrm{e}, \mathrm{V}} / \mathrm{d} x\right)_{\text {notch root }}}
$$

As a second step, the Neuber formula (Equation 5) has to be adjusted for the approximation of the virtual elastic-plastic strain at the interface $\left(\tilde{\varepsilon}_{\mathrm{V}, \text { inter }}\right)$ based on stresses determined by elasticity theory $\left(\varepsilon_{\mathrm{e}, \mathrm{V} \text {,inter }} \mid \sigma_{\mathrm{e}, \mathrm{V} \text {,inter }}\right)$. Therefore the new virtual stress-strain relation of the interface is used as the material model, whereby the calculation of the modified stress includes a different limit load factor $K_{\mathrm{pR}}$, Equation 6. This new factor is a percentage of $K_{\mathrm{p}}$ and depends on the elasticity theoretical stress gradients at the interface as well as the notch root, Equation 7. The formula was determined empirically on the basis of the results of the finite element analyses. The stresses and stress gradients required for these calculations are illustrated in Figure 2.

$$
\begin{aligned}
& \sigma_{\mathrm{e}, \mathrm{V}, \text { inter }} \cdot \varepsilon_{\mathrm{e}, \mathrm{V}, \text { inter }} \cdot\left(\frac{\varepsilon^{*}}{\sigma^{*} / E}\right)=\tilde{\sigma}_{\mathrm{V}, \text { inter }} \cdot \tilde{\varepsilon}_{\mathrm{V}, \text { inter }} \\
& \varepsilon^{*}=\frac{\sigma^{*}}{E}+\left(\frac{\sigma^{*}}{K_{\text {inter }}^{\prime}}\right)^{\frac{1}{n^{\prime}}} \quad \text { with } \quad \sigma^{*}=\frac{\sigma_{\mathrm{e}, \mathrm{V}, \text { inter }}}{K_{\mathrm{pR}}} \\
& K_{\mathrm{pR}}=1+\left(\frac{\left(\mathrm{d} \sigma_{\mathrm{e}, \mathrm{V}} / \mathrm{d} x\right)_{\text {inter }}}{\left(\mathrm{d} \sigma_{\mathrm{e}, \mathrm{V}} / \mathrm{d} x\right)_{\text {notch root }}}\right) \cdot\left(K_{\mathrm{p}}-1\right)
\end{aligned}
$$


The necessity of this modification of Neuber's approximation method can be shown by a simple example: For the specified two-dimensional model with $d=0.5 \mathrm{~mm}$ and $R=2 \mathrm{~mm}$, a finite element analysis without material mismatch $\left(K_{\text {core }}^{\prime}=K_{\text {surf.lay }}^{\prime}\right)$ was performed. By comparing the estimated elastic-plastic strains at the interface of the standard Neuber approximation method as well as of the improved procedure to the FEA results, it is evident that the modification leads to a significantly lower discrepancy, Figure 2. This was confirmed for many different geometry variations.
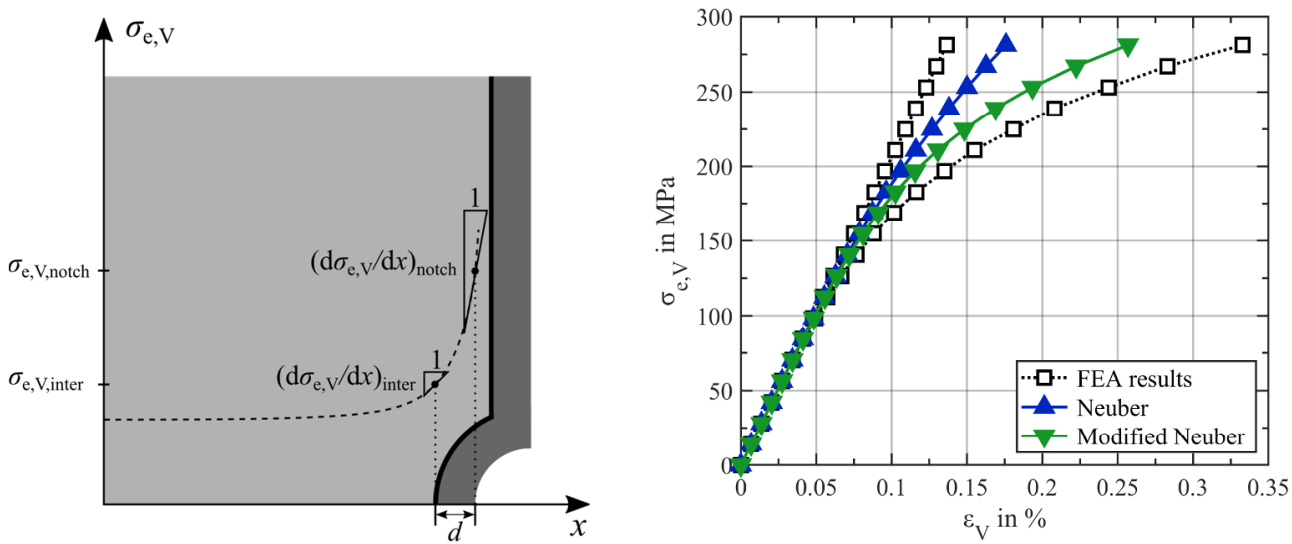

Fig. 2. Sketch of the geometry and stress distribution according to the theory of linear elasticity (left). Example comparison of the approximations of elastic-plastic strains at the interface to FEA (right).

\section{Calculation procedure}

In the following, a six-step algorithm for the approximation of elastic-plastic local strains for both failure-relevant points in surface-hardened notched components is described, as shown schematically in Figure 3. As input data, the results of an elasticity-theoretical finite element analysis for the interface (in Figure 3: Point 1) and the notch root (Point 2) is required. Furthermore the material properties of the core material and the surface layer are mandatory, whereby they are identical for the elastic region.

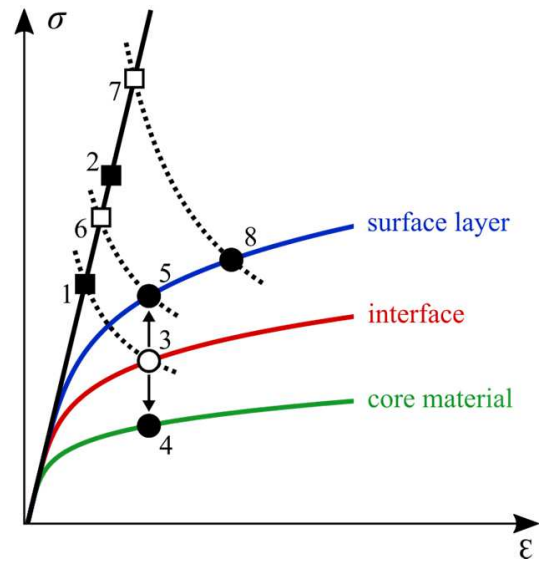

Fig. 3. Schematic illustration of the calculation procedure for the approximation of elastic-plastic local strains at the core-side interface (Point 4) and notch root (Point 8). 


\subsection{Approximation for the interface}

The first step of the calculation procedure is identical to the modified Neuber approximation method previously explained in Section 2.2: Based on the elasticity-theoretical stresses at the interface calculated by FEA, the virtual elastic-plastic strain at the interface is approximated and results in the virtual stresses at the interface (Point 3). Due to the initial assumption of deformation compatibility and uniform equivalent strains on both sides, the virtual elasticplastic strain at the interface corresponds directly to those of the core-side and surface-layerside interface, Equation 8.

$$
\varepsilon_{\mathrm{V}, \text { core_inter }}=\varepsilon_{\mathrm{V}, \text { surf.lay_inter }}=\tilde{\varepsilon}_{\mathrm{V}, \text { inter }}
$$

The stress can be determined by using the corresponding stress-strain relationship and thus provides the first failure-relevant location for the proof of structural durability, the core-side interface (Point 4). Analogously, the stress at the surface-layer-side interface (Point 5) can be calculated with the respective stress-strain relationship.

$$
\begin{aligned}
& \varepsilon_{\mathrm{V}, \text { core_inter }}=\frac{\sigma_{\mathrm{V}, \text { core_inter }}}{E}+\left(\frac{\sigma_{\mathrm{V}, \text { core_inter }}}{K_{\text {core }}^{\prime}}\right)^{\frac{1}{n^{\prime}}} \\
& \varepsilon_{\mathrm{V}, \text { surflay_inter }}=\frac{\sigma_{\mathrm{V}, \text { surf.lay_inter }}}{E}+\left(\frac{\sigma_{\mathrm{V}, \text { surfllay_inter }}}{K_{\text {surf.lay }}^{\prime}}\right)^{\frac{1}{n^{\prime}}}
\end{aligned}
$$

\subsection{Approximation for the notch root}

From the stress and elastic-plastic strain at the interface, surface-layer-side, the pseudo elastic strain at this point is determined by using the modified Neuber approximation method backwards (Point 6). The stresses are larger by a factor than the elasticity-theoretical one for homogeneous structures, since the plastification of the core material has been taken into account, where appropriate.

$$
\begin{aligned}
& \tilde{\sigma}_{\text {e,V,surf.lay_inter }} \cdot \tilde{\varepsilon}_{\text {e,V,surf.lay_inter }} \cdot\left(\frac{\varepsilon^{*}}{\sigma^{*} / E}\right)=\sigma_{\mathrm{V}, \text { surf.lay_inter }} \cdot \varepsilon_{\mathrm{V}, \text { surf.lay_inter }} \\
& \varepsilon^{*}=\frac{\sigma^{*}}{E}+\left(\frac{\sigma^{*}}{K_{\text {surf.lay }}^{\prime}}\right)^{\frac{1}{n^{\prime}}} \quad \text { with } \quad \sigma^{*}=\frac{\tilde{\sigma}_{\mathrm{e}, \mathrm{V}, \text { surfllay_inter }}}{K_{\mathrm{pR}}}
\end{aligned}
$$

The elasticity-theoretical notch root stress is then increased by this particular factor, using Equation 13, and thus results in the pseudo elastic strain in the notch root (Point 7), according to Equation 14.

$$
\begin{aligned}
& \tilde{\sigma}_{\mathrm{e}, \mathrm{V}, \text { notch root }}=\tilde{\sigma}_{\mathrm{e}, \mathrm{V}, \text { surf.lay_inter }} \cdot\left(\frac{\sigma_{\mathrm{e}, \mathrm{V}, \text { notch root }}}{\sigma_{\mathrm{e}, \mathrm{V}, \text { inter }}}\right) \\
& \tilde{\varepsilon}_{\mathrm{e}, \mathrm{V}, \text { notch root }}=\frac{\tilde{\sigma}_{\mathrm{e}, \mathrm{V}, \text { notch root }}}{E}
\end{aligned}
$$

As the final step of the calculation procedure, the standard Neuber formula is applied, this time in conjunction with the stress-strain relationship of the surface layer, to approximate the 
elastic-plastic local strain of the notch root, Equation 15 to 17. A second evaluation of the fatigue strength must be performed for this location (Point 8).

$$
\begin{aligned}
& \tilde{\sigma}_{\mathrm{e}, \mathrm{V}, \text { notch root }} \cdot \tilde{\varepsilon}_{\mathrm{e}, \mathrm{V}, \text { notch root }} \cdot\left(\frac{\varepsilon^{*}}{\sigma^{*} / E}\right)=\sigma_{\mathrm{V}, \text { notch root }} \cdot \varepsilon_{\mathrm{V}, \text { notch root }} \\
& \varepsilon^{*}=\frac{\sigma^{*}}{E}+\left(\frac{\sigma^{*}}{K_{\text {surf.lay }}^{\prime}}\right)^{\frac{1}{n^{\prime}}} \quad \text { with } \quad \sigma^{*}=\frac{\tilde{\sigma}_{\mathrm{e}, \mathrm{V}, \text { notch root }}}{K_{\mathrm{p}}} \\
& \varepsilon_{\mathrm{V}, \text { notch root }}=\frac{\sigma_{\mathrm{V}, \text { notch root }}}{E}+\left(\frac{\sigma_{\mathrm{V}, \text { notch root }}}{K_{\text {surf.lay }}^{\prime}}\right)^{\frac{1}{n^{\prime}}}
\end{aligned}
$$

\subsection{Accuracy}

To validate the accuracy of the new algorithm for surface-hardened notch components, numerous finite element analyses were performed to compare the results of the elastic-plastic local strains at the core-side interface and the notch root with approximated values. Varying the surface layer width $d$, the notch radius $R$ and the material properties of core material and surface layer, this leads to a very high number of different possible combinations. Therefore, only a selection of different geometry variants for one set of material parameters is presented below in order to present the most important conclusions.

In the following investigations, the elastic material properties are identical for the surface layer and the core material, and thus as well for the virtual interface, with a Young's modulus of $E=206000 \mathrm{MPa}$ and a Poisson's ratio of $v=0.3$. Furthermore, the following parameters are defined for characterisation of the elastic-plastic material behaviour: $K_{\text {core }}^{\prime}=886 \mathrm{MPa}$, $K_{\text {surf.lay }}^{\prime}=1664 \mathrm{MPa}$ and $n_{\text {core }}^{\prime}=n_{\text {surf.lay }}^{\prime}=n_{\text {inter }}^{\prime}=0.187$. For the interface, the cyclic strength coefficient $K_{\text {inter }}^{\prime}$ is determined as previously described in Equation 4 . These stressstrain relationships are implemented by the multilinear kinematic hardening model into finite element analyses. The applied load is $S=300 \mathrm{MPa}$ and is increased in 20 substeps.

The following Figures 4 to 6 show the approximated elastic-plastic local strains determined with the new algorithm for the two failure-relevant points. For comparison and validation of the accuracy, the calculated values from finite element analyses are given. The first example, a model with surface layer width $d=0.5 \mathrm{~mm}$ and notch radius $R=2 \mathrm{~mm}$, shows for the coreside interface a decent approximation of the elastic-plastic strains and for the notch root a very accurate approximation, Figure 4 . If the surface layer thickness is doubled to $d=1 \mathrm{~mm}$ for the same notch radius, it can be seen that this results in greater discrepancy in accuracy for both points, but especially for the notch root, Figure 5. For the next example the notch radius is increased to $R=5 \mathrm{~mm}$ while the surface layer thickness $d=2 \mathrm{~mm}$ remains unchanged, Figure 6. It is evident that this has only a minor effect on the accuracy of the approximated elastic-plastic local strains at the core-side interface. For the notch root, this increase obviously leads to a smaller deviation and thus to a better match between the approximation and the FEA solution.

The accuracy of the approximation is therefore directly linked to the geometric variables of surface layer thickness $d$ and notch radius $R$. It is essential to find a compromise that conservatively estimates the resulting stresses at both verification points for all combinations. Further work is ongoing to improve the accuracy of the modified approximation method. 

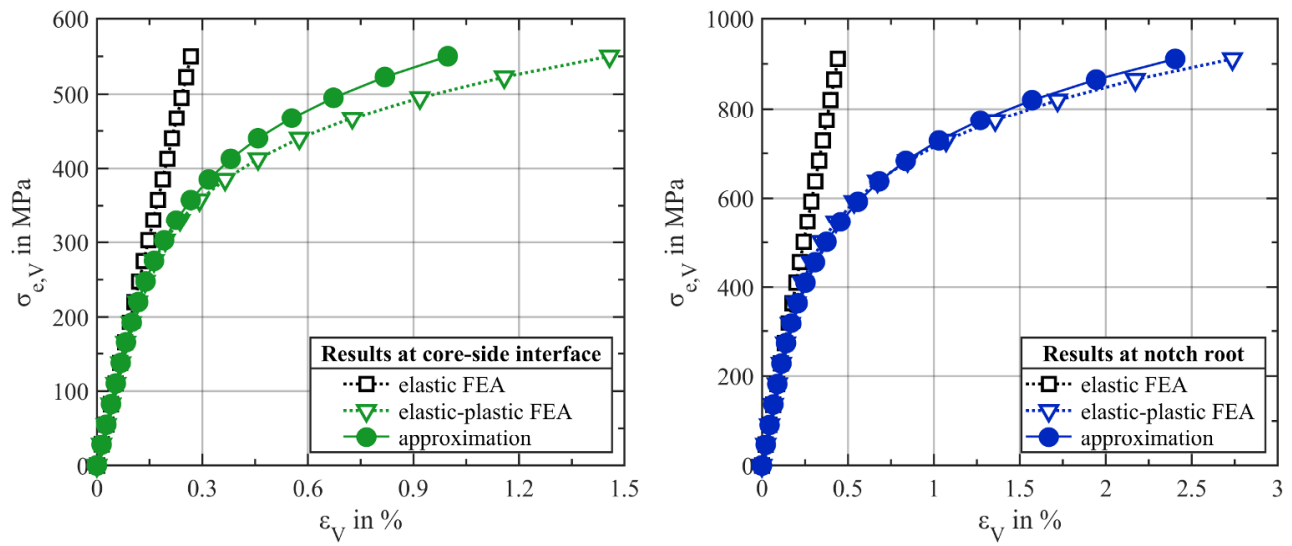

Fig. 4. Comparison of the approximations to FEA results of the core-side interface (left) and the notch root (right) for surface layer width $d=0.5 \mathrm{~mm}$ and notch radius $R=2 \mathrm{~mm}$.
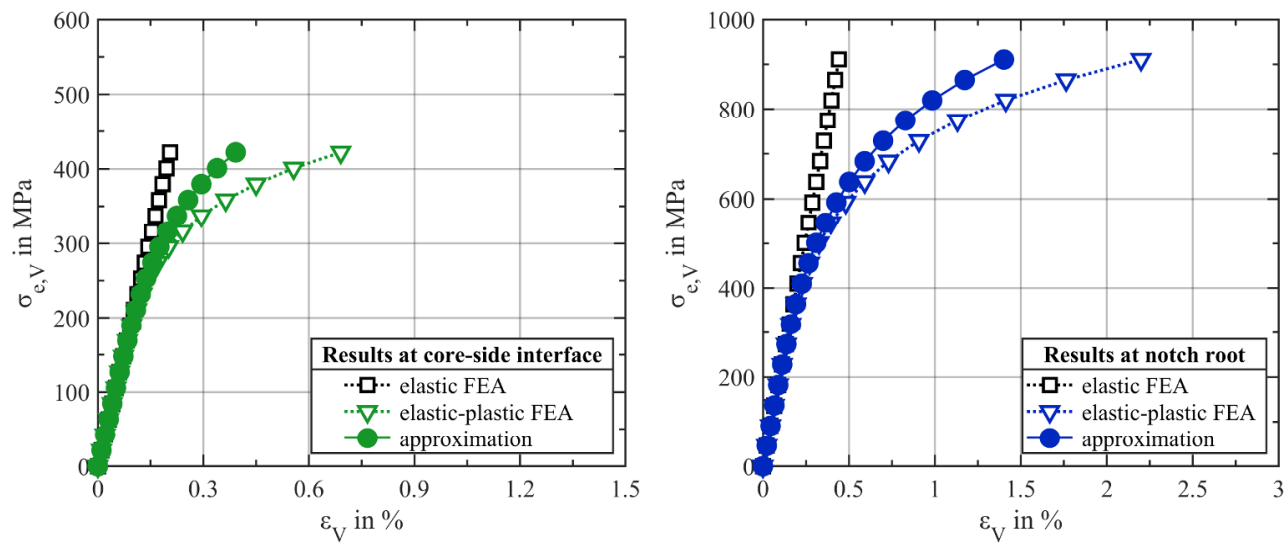

Fig. 5. Comparison of the approximations to FEA results of the core-side interface (left) and the notch root (right) for surface layer width $d=1 \mathrm{~mm}$ and notch radius $R=2 \mathrm{~mm}$.
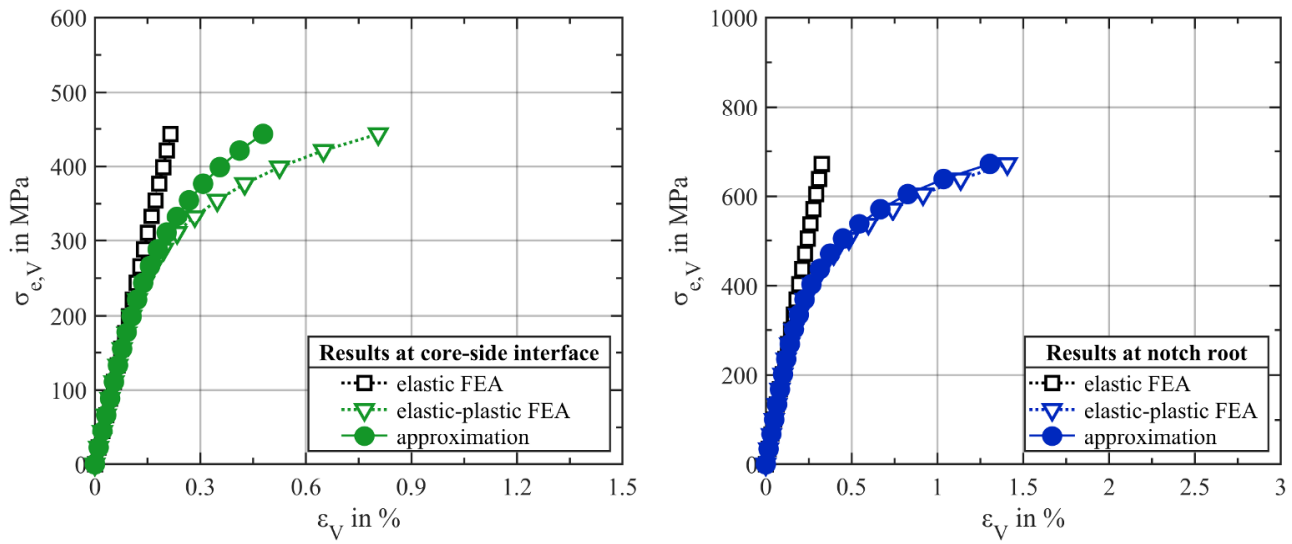

Fig. 6. Comparison of the approximations to FEA results of the core-side interface (left) and the notch root (right) for surface layer width $d=1 \mathrm{~mm}$ and notch radius $R=5 \mathrm{~mm}$. 


\section{Conclusion}

As highly stressed components often require the use of surface layer strengthening technique to ensure their resistance, this leads to a structure with inhomogeneous material properties and thus to two failure-relevant points: The notch root and the interface between the low strength core material and the high strength surface layer. For proofs of structural durability based on the local strain approach, the elastic-plastic strains at these points are estimated with approximation methods based on elasticity-theoretical determined stresses, whereby this is currently only approved for homogeneous components. Therefore, a new algorithm based on a modified variant of Neuber's approximation method was presented here. A surfacehardened component is first simplified to a structure with only two homogeneous areas, core material and surface layer. A virtual stress-strain relationship is introduced for the interface, at which deformation compatibility prevails, taking into account geometry-related influencing variables. For the approximation of the stresses at the core-side interface, the Neuber formula is also adapted by introducing a new limit load factor. The entire calculation procedure starts by estimating the elastic-plastic local strains at the interface, core- and surface-layer-side, based on elasticity-theoretical FEA results at the interface. From this point on the surface-layer-side interface, the pseudo-elastic strains there are again determined with the modified Neuber formula backwards. They are larger by a factor than the elasticitytheoretical ones for homogeneous structures, because the plasticisation of the core material. The elasticity-theoretical notch root stress is increased by exactly this factor and then used for a third application of the Neuber formula to estimate the elastic-plastic local strains of the notch root. By comparing the estimated values with those calculated using FEA, it was possible to show that this method provides a good accuracy despite its simplicity. It can also be seen that the surface layer thickness and the notch radius have a direct effect on the difference between the approximation and the exact calculation. The ongoing research will show how the modified approximation method can be further improved.

This new method for elastic-plastic strains is intended to modify the "FKM Guideline non-linear" and to enhance the algorithms of the local strain approach for components affected by surface layer strengthening techniques. For this purpose, a two-point verification will be implemented, which allows the proof of structural durability for both failure-relevant points of surface-hardened notched components. Furthermore, the consideration of residual stresses will be implemented, which is of particular interest for these components.

The present research is part of the IGF project No. $20745 \mathrm{~N}$ of the Research Association "Forschungskuratorium Maschinenbau e.V." (FKM), which is funded by the AiF as part of the programme for the support of joint industrial research (IGF) by the Federal Ministry for Economic Affairs and Energy on the basis of a resolution of the German Bundestag.

\section{References}

1. T. Seeger, P. Heuler, Ermüdungsverhalten metallischer Werkstoffe, 213-235, (1985)

2. M. Vormwald, T. Seeger, Crack Initiation Life Estimations for Notched Specimens with Residual Stresses Based on Local Strains, Residual Stresses in Science and Technology, 743-750, (1987)

3. M. Fiedler, M. Wächter, I. Varfolomeev, M. Vormwald, A. Esderts, Richtlinie nichtlinear, (2019)

4. H. Neuber, Konstruktion 20, 245-251, (1968)

5. M. Hoffmann, T. Seeger, J. Eng. Mater. Technol. 107(4), 250-254, (1989)

6. T. Seeger, P. Heuler, JTEVA 8, 199-204, (1980) 